\title{
Ocorrência de multiparasitismo em larvas de terceiro ínstar e pupas de Chrysomya megacephala (Fabricius) em condições de campo
}

\author{
Alessandra Ribeiro de Carvalho ${ }^{1,3}$, José Mário d'Almeida ${ }^{2} \&$ Rubens Pinto de Mello
}

\begin{abstract}
${ }^{1}$ Curso de pós-graduação em Biologia Parasitária, Instituto Oswaldo Cruz/ Fundação Oswaldo Cruz. Av. Brasil, 4365, Manguinhos, 21045-900
Rio de Janeiro-RJ. acarvalho@ufla.br

${ }_{2}^{2}$ Instituto de Biologia, Universidade Federal Fluminense (UFF). Outeiro de São João Batista, s/n, 24020-150 Niterói-RJ.

${ }^{3}$ Laboratório de Diptera, Departamento de Entomologia, Instituto Oswaldo Cruz/ Fundação Oswaldo Cruz.
\end{abstract}

\begin{abstract}
Occurrence of multiparasitism in third instar larvae and pupae of Chrysomya megacephala (Fabricius) in field conditions. During research experiment involving parasitism of third instar larvae and pupae of Chrysomya megacephala (Fabricius, 1794) in Rio de Janeiro city, Brazil, it was found $1.83 \%$ of multiparasited pupae in Instituto Oswaldo Cruz (IOC) and 2.16\% in Jardim Zoológico (ZOO). The exposition were conducted weekly from August 1999 to July 2000. The multiparasited pupae contained two Hymenoptera parasitoids species [Tachinaephagus zealandicus Ashmead, 1904 and either Pachycrepoideus vindemiae (Rondani, 1875) or Nasonia vitripennis (Walker, 1836)]. 72.73\% of the multiparasitism occurred at ZOO in July. T. zealandicus and P. vindemiae supported their respective gregarious and solitary natural conditions, respectively, but $N$. vitripennis sometimes behaved as solitary.
\end{abstract}

\begin{abstract}
Keywords. Nasonia vitripennis; Pachycrepoideus vindemiae; synanthropic flies; Tachinaephagus zealandicus.
Resumo. Ocorrência de multiparasitismo em larvas de terceiro ínstar e pupas de Chrysomya megacephala (Fabricius) em condições de campo. Durante experimento de pesquisa envolvendo o parasitismo de larvas de terceiro ínstar e pupas de Chrysomya megacephala (Fabricius, 1794) em dois locais da cidade do Rio de Janeiro, RJ, observou-se a ocorrência de multiparasitismo em $1,83 \%$ das pupas expostas no Instituto Oswaldo Cruz (IOC) e em 2,16\% expostas no Jardim Zoológico (ZOO). O experimento foi conduzido semanalmente de agosto de 1999 a julho de 2000. Em ambos os locais, houve a co-ocorrência de duas espécies por pupa e os parasitóides encontrados foram os himenópteros Tachinaephagus zealandicus Ashmead, 1904, Pachycrepoideus vindemiae (Rondani, 1875) e Nasonia vitripennis (Walker, 1836). 72,73\% do multiparasitismo ocorreu no ZOO em julho de 2000. Em condições de multiparasitismo, T. zealandicus e P. vindemiae mantiveram seu hábito gregário e solitário, respectivamente, mas $N$. vitripennis foi encontrado solitário em algumas pupas.
\end{abstract}

Palavras-Chave. Moscas sinantrópicas; Nasonia vitripennis; Pachycrepoideus vindemiae; Tachinaephagus zealandicus.

Chrysomya megacephala (Fabricius, 1794) é uma mosca de considerável importância em grandes cidades devido ao seu alto grau de sinantropia e ao acúmulo de material orgânico em decomposição nesses locais, resultante do destino inadequado do lixo doméstico e um sistema sanitário ineficaz, principalmente em países subdesenvolvidos. Esta mosca é utilizada como indicadora do intervalo de tempo de morte de cadáveres humanos em Entomologia Forense (Carvalho et al. 2000) e é veiculadora de enteropatógenos como vírus, bactérias e helmintos para humanos e animais (Furlanetto et al. 1984).

Estudos sobre controle biológico de C. megacephala são incipientes. Na Malásia, Sulaiman et al. (1990) encontraram pupas de C. megacephala parasitadas por himenópteros da família Pteromalidae: Spalangia endius Walker, 1839, Spalangia cameroni Perkins, 1910 e Pachycrepoideus vindemiae (Rondani, 1875). Na Coréia do Sul, esta mosca teve como principais parasitóides Spalangia nigroaenea Curtis, 1839, Spalangia nigra Latreille, 1805, Muscidifurax raptor (Girault \& Sanders, 1910) e P. vindemiae (Rueda et al. 1997), ambos himenópteros da família Pteromalidae. Na cidade do Rio de Janeiro, Carvalho et al. (2003) encontraram três espécies de microhimenópteros parasitóides, sendo elas Tachinaephagus zealandicus Ashmead, 1904 (Encyrtidae), $P$. vindemiae e Nasonia vitripennis (Walker, 1836) (Pteromalidae).

A ocorrência de duas espécies concomitantemente no pupário de um mesmo hospedeiro raramente é relatada em resultados de levantamentos de microhimenópteros parasitóides de moscas sinantrópicas, o que pode significar ausência, negligenciamento do fenômeno ou morte de um dos inimigos naturais competidores pois, de acordo com Fisher (1961), quando um inseto é atacado por mais de uma espécie de parasitóide, ocorrerá competição entre elas e algumas vezes somente uma espécie sobrevive. O reconhecimento da presença da segunda espécie é difícil em determinadas fases do seu desenvolvimento e técnicas mais precisas têm sido utilizadas para detectar o multiparasitismo, sendo PCR ("Polymerase Chain Reaction") uma delas (Ratcliffe et al. 2002).

Este artigo tem como objetivo relatar a ocorrência de multiparasitismo entre microhimenópteros parasitóides de larvas de terceiro ínstar e pupas de C. megacephala em condições de campo na cidade do Rio de Janeiro, RJ. 
Tabela I. Número e sexo dos microhimenópteros parasitóides emergidos das pupas de Chrysomya megacephala (Diptera, Calliphoridae) multiparasitadas no campus do Instituto Oswaldo Cruz (IOC)/ FIOCRUZ e no Jardim Zoológico (ZOO), cidade do Rio de Janeiro, RJ, durante o período de agosto de 1999 a julho de 2000.

\begin{tabular}{lccc}
\hline & \multicolumn{3}{c}{ Espécies de Microhimenópteros } \\
\cline { 2 - 4 } Local e data de coleta & $\begin{array}{c}\text { Tachinaephagus } \\
\text { zealandicus }\end{array}$ & $\begin{array}{c}\text { Pachycrepoideus } \\
\text { vindemiae }\end{array}$ & $\begin{array}{c}\text { Nasonia } \\
\text { vitripennis }\end{array}$ \\
\hline 1. IOC: agosto/99 & $17(15 \mathrm{~F}+2 \mathrm{M})^{*}$ & $1(1 \mathrm{~F})$ & - \\
2. IOC: setembro/99 & $11(9 \mathrm{~F}+2 \mathrm{M})$ & $1(1 \mathrm{~F})$ & - \\
3. IOC: outubro/99 & $4(4 \mathrm{~F})$ & $2(1 \mathrm{~F}+1 \mathrm{M})$ & - \\
4. IOC: abril/00 & $10(9 \mathrm{~F}+1 \mathrm{M})$ & - & $1(1 \mathrm{M})$ \\
5. IOC: abril/00 & $13(13 \mathrm{~F})$ & - & $2(2 \mathrm{~F})$ \\
6. IOC: abril/00 & $7(6 \mathrm{~F}+1 \mathrm{M})$ & - & $1(1 \mathrm{M})$ \\
7. ZOO: maio/00 & $13(12 \mathrm{~F}+1 \mathrm{M})$ & - & $18(15 \mathrm{~F}+3 \mathrm{M})$ \\
8. ZOO: maio/00 & $3(2 \mathrm{~F}+1 \mathrm{M})$ & - & - \\
9. ZOO: maio/00 & $6(4 \mathrm{~F}+2 \mathrm{M})$ & $1(1 \mathrm{M})$ & $1(1 \mathrm{~F})$ \\
10.ZOO: junho/00 & $9(6 \mathrm{~F}+3 \mathrm{M})$ & - & $1(1 \mathrm{~F})$ \\
11. ZOO: junho/00 & $7(5 \mathrm{~F}+2 \mathrm{M})$ & - & - \\
12. ZOO: julho/00 & $15(8 \mathrm{~F}+7 \mathrm{M})$ & - & - \\
13. ZOO: julho/00 & $6(4 \mathrm{~F}+2 \mathrm{M})$ & $1(1 \mathrm{~F})$ & - \\
14. ZOO: julho/00 & $11(7 \mathrm{~F}+4 \mathrm{M})$ & $1(1 \mathrm{M})$ & - \\
15. ZOO: julho/00 & $16(9 \mathrm{~F}+7 \mathrm{M})$ & $1(1 \mathrm{M})$ & - \\
16. ZOO: julho/00 & $17(10 \mathrm{~F}+7 \mathrm{M})$ & $1(1 \mathrm{~F})$ & $1(1 \mathrm{M})$ \\
17. ZOO: julho/00 & $18(13 \mathrm{~F}+5 \mathrm{M})$ & $1(1 \mathrm{~F})$ & $6(4 \mathrm{~F}+2 \mathrm{M})$ \\
18. ZOO: julho/00 & - & $1(1 \mathrm{M})$ & $2(2 \mathrm{~F})$ \\
19. ZOO: julho/00 & - & $1(1 \mathrm{M})$ & $1(1 \mathrm{M})$ \\
20. ZOO: julho/00 & - & $1(1 \mathrm{M})$ & $1(1 \mathrm{~F})$ \\
21. ZOO: julho/00 & - & $1(1 \mathrm{~F})$ & \\
22. ZOO: julho/00 & - & &
\end{tabular}

*: Foram utilizadas as letras F e M para designar fêmeas e machos respectivamente.

\section{MATERIAL EMÉTODOS}

Larvas de terceiro ínstar de C. megacephala foram expostas ao parasitismo durante o período de agosto de 1999 a julho de 2000, permanecendo por sete dias próximas a lixeiras do campus do Instituto Oswaldo Cruz (IOC)/Fiocruz e do Jardim Zoológico (ZOO), ambos localizados em bairros residenciais da zona norte da cidade do Rio de Janeiro. As larvas encontravam-se junto ao seu substrato de criação (carne bovina moída putrefata) e sobre uma camada de areia, onde ocorreu a pupação. Após este período, as pupas foram levadas para laboratório, individualizadas em cápsulas de gelatina e armazenadas em câmara climática a $27 \pm 2{ }^{\circ} \mathrm{C}$ por 35 dias. Os pupários foram dissecados e os parasitóides, emergidos ou não, foram contados e identificados.

\section{RESULTADOS E DISCUSSÃO}

Foram encontradas 22 pupas de C. megacephala multiparasitadas, o que equivaleu a $1,83 \%$ do total de pupários com parasitóides no IOC e 2,16\% no ZOO, ocorrendo, no máximo, duas espécies de parasitóides diferentes por pupário.
As espécies de parasitóides encontradas foram Tachinaephagus zealandicus, Pachycrepoideus vindemiae e Nasonia vitripennis (Tabela I). T. zealandicus é uma espécie endoparasita de larvas de moscas e de hábito gregário, enquanto $P$. vindemiae tem hábito solitário e parasita pupas. $N$. vitripennis é um ectoparasitóide gregário de pupas de moscas.

O multiparasitismo foi mais marcante no ZOO $(72,73 \%)$, destacando o mês de julho de 2000 (Tabela I), quando ocorreu o pico de parasitismo por $T$. zealandicus e $P$. vindemiae (Carvalho et al. prelo), reflexo do acúmulo de material orgânico em decomposição nas lixeiras e conseqüente aumento de seus vários hospedeiros.

O número de parasitóides emergidos por pupa multiparasitada, bem como a proporção de machos e fêmeas de $T$. zealandicus e $P$. vindemiae, não sofreu alteração com a presença de outra espécie no mesmo hospedeiro, uma vez que essas espécies mantiveram, respectivamente, seu hábito gregário e solitário, embora $P$. vindemiae tenha mostrado um caso de superparasitismo (Tabela I, número 3). Todavia, o número de $N$. vitripennis por hospedeiro foi modificado, tendo sido encontrado um espécime por pupa multiparasitada, apesar 
de seu hábito gregário, com exceção de três coletas do ZOO (Tabela I, números 8, 19 e 20) e uma coleta no IOC (Tabela I, número 6).

A maior emergência de parasitóides da espécie $T$. zealandicus em relação às demais espécies é, provavelmente, resultado do hábito de parasitar larvas e ter uma localização diferenciada em relação às demais espécies (endoparasita). $N$. vitripennis e $P$. vindemiae, ao contrário, são ectoparasitóides de pupas, e por isso pode haver competição por alimento e espaço durante o desenvolvimento das larvas, ocasionando a deficiência de oxigênio e escassez de nutrientes, além de secreção de toxinas (Utsunomiya \& Ikabuchi 2002).

Até 1939, a ocorrência concomitante de $P$. vindemiae e outro parasitóide era pouco conhecida (Crandell 1939). Segundo o autor, o multiparasitismo não era observado porque $P$. vindemiae consegue eliminar a segunda espécie, devido à aparente vantagem de sua posição externa no hospedeiro e posição interna de outros parasitas a ele associados. Quando $P$. vindemiae multiparasitou um hospedeiro junto com $M$. raptor, outro parasita externo, mostrou-se incapaz de completar seu desenvolvimento. Segundo revisão de Wylie (1972), em adição às características específicas inatas, fatores extrínsecos, principalmente o momento do ataque ao hospedeiro pela espécie competidora, afetam o resultado da competição larval interespecífica.

$N$. vitripennis não mostrou-se um bom agente de controle biológico quando comparado com as demais espécies de parasitóides coletadas na cidade do Rio de Janeiro, pois apresentou a menor freqüência, teve ocorrência acidental e seu pico populacional ocorreu quando o parasitismo por outras espécies foi igual a zero (Carvalho et al. prelo). Entretanto, mostrou capacidade de sobreviver ao multiparasitismo com $T$. zealandicus e $P$. vindemiae. Em experimento realizado por Toyama \& Ikeda (1980), $N$. vitripennis foi o segundo parasitóide mais comum encontrado em multiparasitismo de Calliphoridae. Segundo Wylie (1972), a superioridade de $N$. vitripennis em relação a algumas espécies (Muscidifurax zaraptor Kogan \& Legner, 1970 e S. cameroni) advém do seu rápido desenvolvimento e completa utilização do hospedeiro, causando a morte de seu competidor.

\section{REFERÊNCIAS}

Carvalho, L. M. L.; P. J. Thyssen; A. X. Linhares \& F. A. B. Palhares. 2000. A checklist of arthropods associated with pig carrion and human corpes in southeastern Brazil. Memórias do Instituto Oswaldo Cruz 95: 135-138.

Carvalho, A. R.; R. P. Mello \& J. M. d'Almeida. 2003. Microhimenópteros parasitóides de Chrysomya megacephala. Revista de Saúde Pública 37: 810-812.

Carvalho, A. R.; R. P. Mello \& J. M. d'Almeida. 2005. Dinâmica populacional e potencial de parasitismo dos microhimenópteros parasitóides de Chrysomya megacephala (Fabricius, 1794) (Diptera: Calliphoridae), na cidade do Rio de Janeiro, RJ. Revista Brasileira de Entomologia 99: 118-122.

Crandell, H. A. 1939. The biology of Packycrepoideus dubius Ashmead (Hymenoptera), a Pteromalidae parasite of Piophila casei Linneu (Diptera). Annals of Entomological Society of America 32 : 632-654.

Fisher, R. C. 1961. A study in insect multiparasitism. The Journal of Experimental Biology 38: 267-275.

Furlanetto, S. M. P.; M. L. C. Campos; C. M. Hársi; G. M. Buralli \& G. K. Ishihata. 1984. Microrganismos enteropatogênicos em moscas africanas pertencentes ao gênero Chrysomyia (Diptera, Calliphoridae) no Brasil. Revista de Microbiologia 15: 170 174.

Ratcliffe, S. T.; C. J. J. Robertson; G. A. Bollero \& R. A. Weinzierl. 2002. Assessment of parasitism of house fly and stable fly (Diptera: Muscidae) pupae by pteromalid (Hymenoptera: Pteromalidae) parasitoids using a polymerase chain reaction assay. Journal of Medical Entomology 39: 52-60.

Rueda, L. M.; P. U. Roh \& J. L. Ryu. 1997. Pupal parasitoids (Hymenoptera: Pteromalidae) of filth flies (Diptera: Muscidae, Calliphoridae) breeding in refuse and poultry and livestock manure in South Korea. Journal of Medical Entomological 34: 82-85.

Sulaiman, S.; B. Omar; S. Omar; J. Jefferey; I. Ghauth \& V. Busparani. 1990. Survey of microhymenoptera (Hymenoptera: Chalcidoidea) parasitizing filth flies (Diptera: Muscidae, Calliphoridae) breeding in refuse and poultry farms in Peninsular Malaysia. Journal of Medical Entomology 27: 851-855.

Toyama, G. M. \& J. K. Ikeda. 1980. Parasites as the cause of high incidence of non-viable fly puparia at animal farms. Proceedings Hawaiian Entomological Society 23: 293-299.

Utsunomiya, A. \& Ikabuchi, K. 2002. Interespecific competition between the polyembryonic wasp Copidosoma floridanum and the gregarious endoparasitoid Glyptapanteles pallipes. Entomologia Experimentals et Applicata 104: 353-362.

Wylie, H. G. 1972. Larval competition among three hymenopterous parasite species on multiparasitized housefly (Diptera) pupae. The Canadian Entomologist 104: 1181-1190.

Recebido em 05.III.2004; aceito em 07.VI.2004 